\title{
Recharging and transportation scheduling for electric vehicle battery under the swapping mode
}

\author{
Huang, A.Q. ${ }^{a}{ }^{*}$, Zhang, Y.Q. ${ }^{a}$, He, Z.F. ${ }^{b,}{ }^{*}$, Hua, G.W. ${ }^{a}$, Shi, X.L. ${ }^{a}$ \\ aschool of Economics and Management, Beijing Jiaotong University, Beijing, P.R. China \\ ${ }^{\mathrm{b}}$ Beijing Wuzi University, Beijing, P.R. China
}

\begin{abstract}
A B S T R A C T
Electric vehicle battery recharging on the swapping mode has grown up as an important option other than the plug-in recharging mode in China, given that several auto giants have been dedicated in constructing their battery swapping systems. However, the lack of effective operational methods on battery recharging and transportation scheduling has aroused a big challenge on the practical application of the swapping mode, which enables the necessity of our work. This study proposes a joint optimization model of recharging and scheduling of electric vehicle batteries with a dynamic electricity price system which is able to identify the optimal charging arrangement (the recharging time and the quantity of recharging batteries) as well as the optimal transportation arrangement (the transportation time and the quantity of transporting batteries). For the validation purpose, a numerical study is implemented based on dynamic electricity prices in Beijing. A sensitivity analysis of parameters is carried out to increase the robustness and provide more managerial insights of the model.
\end{abstract}

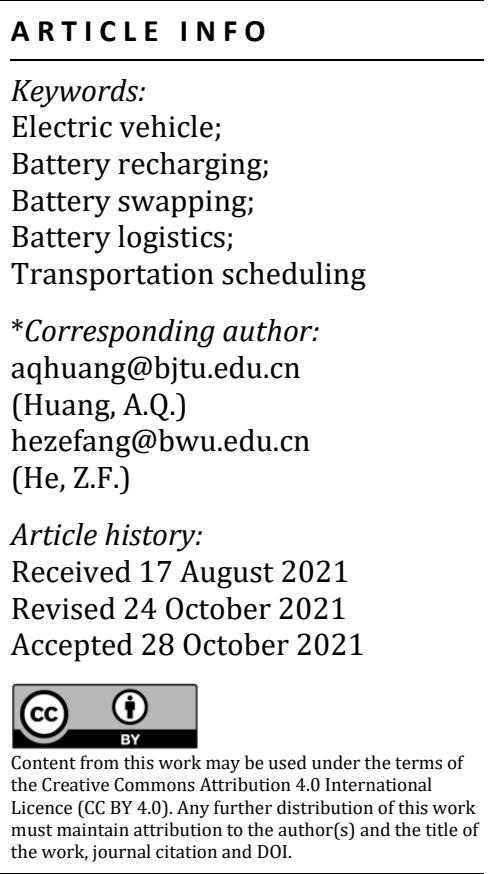

\section{References}

[1] Jie, W., Yang, J., Zhang, M., Huang, Y. (2019). The two-echelon capacitated electric vehicle routing problem with battery swapping stations: Formulation and efficient methodology, European Journal of Operational Research, Vol. 272, No. 3, 879-904, doi: 10.1016/i.ejor.2018.07.002.

[2] Su, W., Eichi, H., Zeng, W., Chow, M.-Y. (2012). A survey on the electrification of transportation in a smart grid environment, IEEE Transactions on Industrial Informatics, Vol. 8, No. 1, 1-10, doi: 10.1109/TII.2011.2172454.

[3] Gong, D., Tang, M., Liu, S., Xue, G., Wang, L. (2019). Achieving sustainable transport through resource scheduling: A case study for electric vehicle charging stations, Advances in Production Engineering \& Management, Vol. 14, No. 1, 65-79, doi: 10.14743/apem2019.1.312.

[4] Barmaki, R., Ilkhani, M., Salehpour, S. (2016). Investigation of energy usage and emissions on plug-in and hybrid electric vehicle, Tehnički Vjesnik - Technical Gazette, Vol. 23, No. 3, 899-906, doi: 10.17559/TV-20140928112 $\underline{417 .}$

[5] Pirbadali-Somarin, A., Peyghambarzadeh, S.M. (2020). Air pollution by heavy metals from petrochemical incinerators: Measurement and dispersion modelling, Environmental Engineering and Management Journal, Vol. 19, No. 3, 379-390, doi: 10.30638/eemi.2020.036.

[6] Wang, G., Xu, Z., Wen, F., Wong, K.P. (2013). Traffic-constrained multiobjective planning of electric-vehicle charging stations, IEEE Transactions on Power Delivery, Vol. 28, No. 4, 2363-2372, doi: 10.1109/TPWRD.2013.2269 142. 
[7] Wang, G., Zhao, J., Wen, F., Xue, Y., Ledwich, G. (2015). Dispatch strategy of PHEVs to mitigate selected patterns of seasonally varying outputs from renewable generation, IEEE Transactions on Smart Grid, Vol. 6, No. 2, 627-639, doi: 10.1109/TSG.2014.2364235.

[8] Tao, Y., Huang, M., Chen, Y., Yang, L. (2020). Orderly charging strategy of battery electric vehicle driven by realworld driving data, Energy, Vol. 193, Article No. 116806, doi: 10.1016/i.energy.2019.116806.

[9] Pieltain Fernández, L., Gómez San Román, T., Cossent, R., Mateo Domingo, C., Frías, P. (2011). Assessment of the impact of plug-in electric vehicles on distribution networks, IEEE Transactions on Power Systems, Vol. 26, No. 1, 206-213, doi: 10.1109/TPWRS.2010.2049133.

[10] Clement-Nyns, K., Haesen, E., Driesen, J. (2010). The impact of charging plug-in hybrid electric vehicles on a residential distribution grid, IEEE Transactions on Power Systems, Vol. 25, No. 1, 371-380, doi: 10.1109/TPWRS. 2009.2036481.

[11] Zhang, X., Wang, G. (2016). Optimal dispatch of electric vehicle batteries between battery swapping stations and charging stations, In: Proceedings of 2016 IEEE Power and Energy Society General Meeting (PESGM), Boston, Massachusetts, USA, 1-5, doi: 10.1109/PESGM.2016.7741893.

[12] Tang, M., Gong, D., Liu, S., Lu, X. (2017). Finding key factors affecting the locations of electric vehicle charging stations: A simulation and ANOVA approach, International Journal of Simulation Modelling, Vol. 16, No. 3, 541554, doi: 10.2507/IJSIMM16(3)C015.

[13] Stüdli, S., Griggs, W., Crisostomi, E., Shorten, R. (2014). On optimality criteria for reverse charging of electric vehicles, IEEE Transactions on Intelligent Transportation Systems, Vol. 15, No. 1, 451-456, doi: 10.1109/TITS. 2013.2271953.

[14] Deilami, S., Masoum, A.S., Moses, P.S., Masoum, M.A.S. (2011). Real-time coordination of plug-in electric vehicle charging in smart grids to minimize power losses and improve voltage profile, IEEE Transactions on Smart Grid, Vol. 2, No. 3, 456-467, doi: 10.1109/TSG.2011.2159816.

[15] Qiu, D., Yang, H., Lai, M. (2018). Optimal bidding strategy and EV charging management of intelligent regional aggregators based on CVaR method, Systems Engineering - Theory and Practice, Vol. 38, No. 8, 1994-2005, doi: 10.12011/1000-6788(2018)08-1994-12.

[16] Zheng, Y., Dong, Z.Y., Xu, Y., Meng, K., Zhao, J.H., Qiu, J. (2014). Electric vehicle battery charging/swap stations in distribution systems: Comparison study and optimal planning, IEEE Transactions on Power Systems, Vol. 29, No. 1, 221-229, doi: 10.1109/TPWRS.2013.2278852.

[17] Kang, Q., Wang, J.-B., Zhou, M.-C., Ammari, A.C. (2016). Centralized charging strategy and scheduling algorithm for electric vehicles under a battery swapping scenario, IEEE Transactions on Intelligent Transportation Systems, Vol. 17, No. 3, 659-669, doi: 10.1109/TITS.2015.2487323.

[18] Zheng, J., Xie, T., Liu, F., Wang, W., Du, P., Han, Y. (2017). Electric vehicle battery swapping station coordinated charging dispatch method based on CS algorithm, In: Proceedings of 2017 IEEE 3rd Information Technology and Mechatronics Engineering Conference (ITOEC), Chongqing, China, 150-154, doi: 10.1109/ITOEC.2017.8122400.

[19] Yang, S., Yao, J., Kang, T., Zhu, X. (2014). Dynamic operation model of the battery swapping station for EV (electric vehicle) in electricity market, Energy, Vol. 65, 544-549, doi: 10.1016/i.energy.2013.11.010.

[20] Song, Y., Li, J., Ji, G., Xue, Z. (2016). Study on the typical mode of EV charging and battery swap infrastructure interconnecting to power grid, In: Proceedings of 2016 China International Conference on Electricity Distribution (CICED), Xi'an, China, 1-4, doi: 10.1109/CICED.2016.7576066.

[21] Rao, R., Zhang, X., Xie, J., Ju, L. (2015). Optimizing electric vehicle users' charging behavior in battery swapping mode, Applied Energy, Vol. 155, 547-559, doi: 10.1016/j.apenergy.2015.05.125.

[22] Wang, T., Ling, Y., Liu, J., Han, J., Chen, L., Zhao, H., Feng, S., Han, T. (2018). Integrated optimal configuration of electric vehicle charging and battery-swapping station based on ordered charging strategy, In: Proceedings of 2018 China International Conference on Electricity Distribution (CICED), Tianjin, China, 2237-2241, doi: 10.1109/CICED.2018.8592420.

[23] Infante, W.F., Ma, J., Chi, Y. (2016). Operational strategy and load profile sensitivity analysis for an electric vehicle battery swapping station, In: Proceedings of 2016 IEEE International Conference on Power System Technology (POWERCON), Wollongong, New South Wales, Australia, 1-6, doi: 10.1109/POWERCON.2016.7753877.

[24] Sarker, M.R., Pandžić, H., Ortega-Vazquez, M.A. (2015). Optimal operation and services scheduling for an electric vehicle battery swapping station, IEEE Transactions on Power Systems, Vol. 30, No. 2, 901-910, doi: 10.1109/ TPWRS.2014.2331560.

[25] Wang, Y., Yang, Y., Zhang, N., Huang, M. (2017). An integrated optimization model of charging station/batteryswap station/energy storage system considering uncertainty, In: Proceedings of 2017 IEEE International Conference on Energy Internet (ICEI), Beijing, China, 77-82, doi: 10.1109/ICEI.2017.21. 


\section{APEM}

\title{
Načrtovanje polnjenja in transporta zamenljivih baterij za električna vozila
}

\author{
Huang, A.Q. ${ }^{a}{ }^{*}$, Zhang, Y.Q. ${ }^{a}$, He, Z.F. ${ }^{b}{ }^{*}$, Hua, G.W. ${ }^{a}$, Shi, X.L. ${ }^{a}$ \\ aSchool of Economics and Management, Beijing Jiaotong University, Beijing, P.R. China

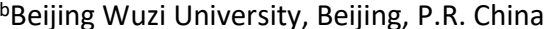

\section{POVZETEK}

Glede na to, da je na Kitajskem več avtomobilskih velikanov gradilo svoje sisteme zamenjave baterije, je postala zamenjava baterije električnega vozila pomembna možnost poleg načina polnjenja baterije prek vtičnika. Vendar pa je pomanjkanje učinkovitih operativnih metod polnjenja in transporta baterij povzročilo velik izziv pri praktični uporabi tehnike zamenjave baterij, kar potrjuje nujnost našega dela. Ta študija predlaga skupni optimizacijski model polnjenja in razporejanja baterij za električna vozila z dinamičnim sistemom cen električne energije, ki je sposoben prepoznati optimalno ureditev polnjenja (čas polnjenja in število baterij za ponovno polnjenje) ter optimalno ureditev transporta (transportni čas in število transportiranih baterij). Za validacijo modela je izvedena numerična študija, ki temelji na dinamičnih cenah električne energije v Pekingu. Da se poveča robustnost in zagotovi boljši vpogled v model je izvedena je občutljivostna analiza.

\section{PODATKI O ČLANKU}

Ključne besede:

Električno vozilo;

Polnjenje baterije;

Zamenjava baterije;

Logistika baterij;

Načrtovanje prevoza

*Kontaktna oseba:

aqhuang@bjtu.edu.cn

(Huang, A.Q.)

hezefang@bwu.edu.cn

(He, Z.F.)

Zgodovina članka:

Prejet 17. avgusta 2021

Popravljen 24. oktobra 2021

Sprejet 28. oktobra 2021

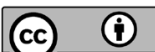

Crom this work may be used under the terms of the Creative Commons Attribution 4.0 International

Licence (CC BY 4.0). Any further distribution of this work must maintain attribution to the author(s) and the title of the work, journal citation and DOI. 